\title{
Azimuthal anisotropies and initial-state fluctuations from SPS to LHC energies
}

\author{
Jovan Milosevic on behalf of the CERES/NA45 and CMS Collaboration ${ }^{1, \star}$ \\ ${ }^{1}$ Vinca Institute of Nuclear Sciences, M. Petrovica Alasa 12-14, 11001 Belgrade, Serbia
}

\begin{abstract}
The $\mathrm{v}_{3}$ coefficient, obtained using the $\mathrm{PbAu}$ data from the CERES detector at the top SPS energy of $\sqrt{s_{\mathrm{NN}}}=17.3 \mathrm{GeV}$, is presented. The $\mathrm{v}_{2}$ is measured over a $\mathrm{p}_{\mathrm{T}}$ range up to $100 \mathrm{GeV} / \mathrm{c}$ in $\mathrm{PbPb}$ collisions collected with the CMS detector. The $\mathrm{v}_{2}\{2\}$ of charged and strange particles emitted in pp collisions shows a mass ordering effect. The $\mathrm{v}_{2}\{4\}$ and $\mathrm{v}_{2}\{6\}$ are comparable to the $\mathrm{v}_{2}\{2\}$, and thus supports the collective nature of the long-range correlations in high-multiplicity pp collisions at $13 \mathrm{TeV}$. Principle Component Analysis (PCA) of two-particle harmonics $\left(\mathrm{V}_{\mathrm{n} \Delta}\right)$ is studied in $\mathrm{PbPb}$ and high-multiplicity $\mathrm{pPb}$ collisions at the LHC. The factorization breaking of the $\mathrm{V}_{\mathrm{n} \Delta}$ can be attributed to the effect of initial-state fluctuations. Using a PCA, the $\mathrm{V}_{\mathrm{n} \Delta}$ are characterized through the leading and sub-leading modes. The leading modes are essentially equivalent to the $\mathrm{v}_{\mathrm{n}}\{2\}$. The sub-leading modes represent the largest sources of factorization breaking.
\end{abstract}

\section{Introduction}

One of main features of Quark Gluon Plasma is its hydrodynamic behavior characterized by $\mathrm{v}_{\mathrm{n}}$ coefficients extracted using methods like two- and many-particle correlations [1], the scalar product (SP) $[2,3]$ and the Lee-Yang Zero (LYZ) $[4,5]$. The CMS collaboration measured $\mathrm{v}_{2}$ coefficient in $\mathrm{PbPb}$ collisions over a wide $\mathrm{p}_{\mathrm{T}}$ range up to $100 \mathrm{GeV} / \mathrm{c}$ [6]. This measurement at high- $\mathrm{p}_{\mathrm{T}}$ is complementary to the nuclear modification factor $\left(\mathrm{R}_{\mathrm{AA}}\right)$ measurements. The long-range $(|\Delta \eta|>2)$ correlation known as the ridge is observed not only in $\mathrm{PbPb}$ collisions [7], but also in high-multiplicity $\mathrm{pPb}$ collisions [8] and even in smallest system formed in high-multiplicity pp collisions $[9,10]$ giving a hint that the ridge could have a hydrodynamic origin. To support it, the correlations among four or more charged particles are studied in $\mathrm{pPb}$ collisions at $\sqrt{s_{N N}}=5.02 \mathrm{TeV}$ [11] and in pp collisions at $\sqrt{s}$ $=5,7$ and $13 \mathrm{TeV}$ [12]. The two-particle correlations between strange $\left(\mathrm{K}_{\mathrm{S}}^{0}\right.$ or $\left.\Lambda / \bar{\Lambda}\right)$ and charged particles [13] also revealed similar ridge structures, and the extracted $\mathrm{v}_{2}$, scaled to the number of constituent quarks $\left(\mathrm{n}_{\mathrm{q}}\right)$ shows that hydrodynamic behavior happens on the partonic level. It is shown in $[14,15]$ that even if the hydrodynamics is the only source of the long-range correlations, the initialstate fluctuations makes the event plane angle, $\Psi_{\mathrm{n}}$, dependent on both, $\mathrm{p}_{\mathrm{T}}$ and $\eta$. This leads to factorization breaking of the $V_{n \Delta}$ into a product of single-particle anisotropies $v_{n}[16]$. A PCA [17, 18] is applied on $\mathrm{V}_{\mathrm{n} \Delta}$ coefficients constructed from 2.76 TeV PbPb collisions and 5.02 TeV high-multiplicity $\mathrm{pPb}$ collisions [19]. Within the PCA approach, the $\mathrm{V}_{\mathrm{n} \Delta}$ coefficients are characterized through leading and sub-leading mode terms. The leading modes are essentially equivalent to the $\mathrm{v}_{\mathrm{n}}$ harmonics

^e-mail: Jovan.Milosevic@cern.ch 
extracted from two-particle correlations. The sub-leading modes represent the largest sources of factorization breaking. In the context of hydrodynamic models, they are a direct consequence of the initial-state fluctuations.

\section{Experiment and data used}

About $30 \mathrm{M}$ of central PbAu collisions at the top SPS energy were collected. CERES detector worked in $0.5 \mathrm{~T}$ magnetic filed which enable precise $\mathrm{p}_{\mathrm{T}}$ measurement. The $\eta$ coverage close to mid-rapidity, together with a full azimuthal $(\phi)$ coverage enables flow study. Negative pions, identified using the differential energy loss $\mathrm{dE} / \mathrm{dx}$ in the Time Projection Chamber (TPC) are used in this analysis.

The CMS tracker detector is surrounded with a super-conducting solenoid producing 3.8 $\mathrm{T}$ magnetic field which enable precise $\mathrm{p}_{\mathrm{T}}$ measurements above $0.3 \mathrm{GeV} / \mathrm{c}$ [20]. The $\mathrm{PbPb}$ and $\mathrm{pPb}$ collisions at the LHC energies of $\sqrt{s_{N N}}=2.76 \mathrm{TeV}$ and $5.02 \mathrm{TeV}$ with integrated luminosities of $160 \mu \mathrm{b}^{-1}$ and $35 \mathrm{nb}^{-1}$, respectively, were collected. The data sets for pp collisions at $\sqrt{s}=5,7$ and $13 \mathrm{TeV}$ have integrated luminosities of $1.0,6.2$ and $0.7 \mathrm{pb}^{-1}$ respectively. A wide pseudorapidity coverage of $|\eta|<2.5$ for the tracker and $2.9<|\eta|<5.2$ for the hadronic forward (HF) calorimeters together with a full azimuthal coverage excellently suites for studying the long-range flow correlations.

\section{Results}

In figure 1, the comparison between the hydrosolver+UrQMD [21] model predictions and CERES $\mathrm{v}_{3}\left(\mathrm{p}_{\mathrm{T}}\right)$ measurement of negative pions in PbAu collisions at $\sqrt{s_{N N}}=17.3 \mathrm{GeV}$ [22] is shown. The predictions of this model are in a good agreement with the experimental results, except in the $\mathrm{p}_{\mathrm{T}}$ region between 0.3 and $0.6 \mathrm{GeV} / \mathrm{c}$ where the model slightly underpredicts the experimental data. The $\mathrm{v}_{3}\left(\mathrm{p}_{\mathrm{T}}\right)$ at the top SPS energy reach only about one half of the corresponding values at the top RHIC and LHC energies. The $\mathrm{p}_{\mathrm{T}}$ integrated $\mathrm{v}_{3}$ value is in an excellent agreement with the STAR $\mathrm{v}_{3}$ value measured at 19.6 GeV [23]. Within the analyzed range, the CERES $v_{3}$ does not show centrality dependence [22].

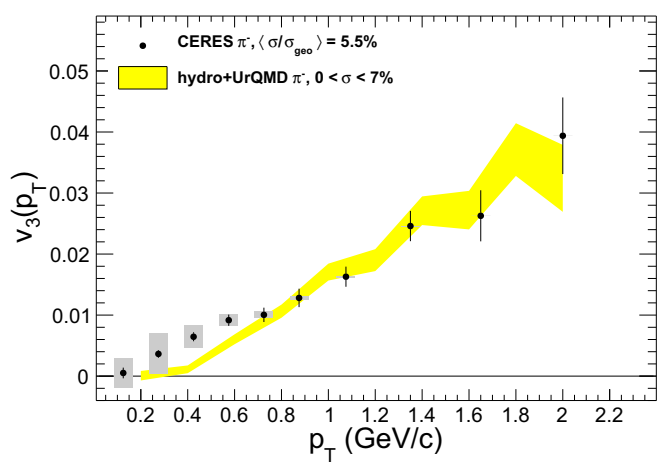

Figure 1. The negative pion $\mathrm{v}_{3}\left(\mathrm{p}_{\mathrm{T}}\right)$ measured in $\mathrm{PbAu}$ collisions at $\sqrt{s_{\mathrm{NN}}}=17.3 \mathrm{GeV}$ compared with hydrosolver+UrQMD model predictions [22]. Statistical uncertainties are represented with the error bars, while systematic ones are indicated by gray rectangles. Statistical errors of the model predictions are shown as yellow band.

The CMS showed in [6] that the $v_{3}$ is consistent with zero while the positive $v_{2}$ persists up to very high- $\mathrm{p}_{\mathrm{T}}$ of nearly $100 \mathrm{GeV} / \mathrm{c}$. To further explore the nature of the $\mathrm{v}_{2}\left(\mathrm{p}_{\mathrm{T}}\right)$ dependence, the $\mathrm{v}_{2}$ values are also calculated using the 4-, 6- and 8- particle cumulant analyses. The obtained results are shown in figure 2 . In the low- $\mathrm{p}_{\mathrm{T}}$ regions, the results show the usual hydrodynamic shape with $\mathrm{v}_{2}\{\mathrm{SP}\}>\mathrm{v}_{2}\{4\} \approx \mathrm{v}_{2}\{6\} \approx \mathrm{v}_{2}\{8\}$. At high- $\mathrm{p}_{\mathrm{T}}$, results tend to converge to a unique distribution. Such observed collectivity at high- $\mathrm{p}_{\mathrm{T}}$ is likely to be related to the jet quenching phenomena and the pathlength dependence of the parton energy loss. 


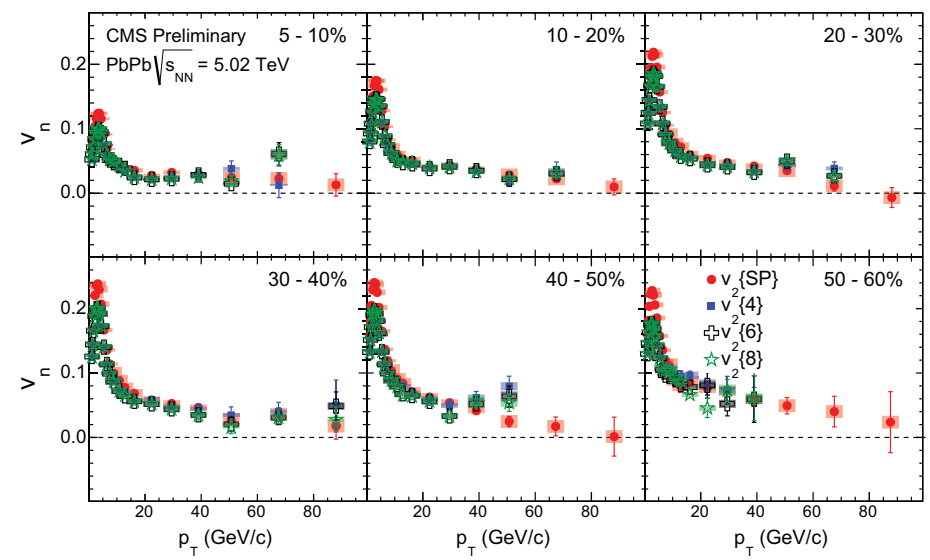

Figure 2. The $\mathrm{SP} \quad \mathrm{v}_{2}$ results compared to those from cumulant method as a function of $\mathrm{p}_{\mathrm{T}}$ over wide centrality range in $5.02 \mathrm{TeV} \mathrm{PbPb}$ collisions [6]. The systematic uncertainties are denoted with shaded boxes.

The $\mathrm{v}_{2}$ coefficient in pp collisions at $13 \mathrm{TeV}$ is studied using the two-particle correlation method as well as cumulant of fourth and sixth order [12]. The $\mathrm{v}_{2}$, as a function of charged particle multiplicity, are shown in the left panel of figure 3. In the high multiplicity region, the results are consistent with each other. Also, the ordering of the pp results obtained from different methods is similar to what was observed in $\mathrm{pPb}$ (middle panel) and $\mathrm{PbPb}$ (right panel) collisions [11]: the relation $v_{2}\{2\} \geq v_{2}\{4\} \approx v_{2}\{6\}$ in all three colliding systems is an evidence of a collective behavior.

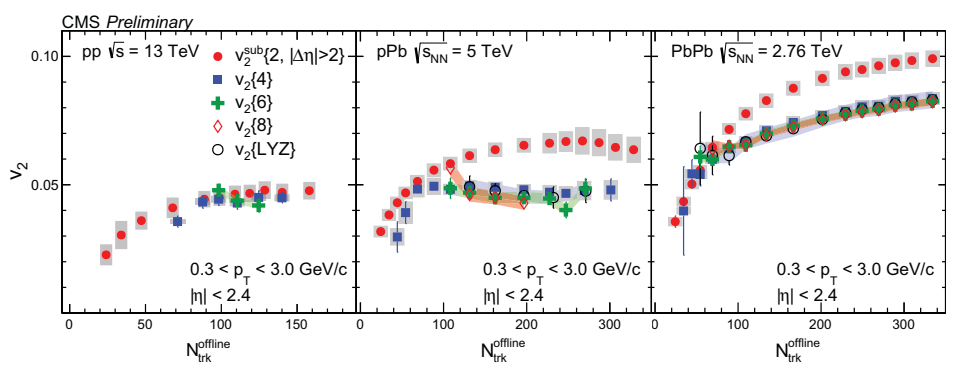

Figure 3. Left: The $v_{2}\{2\}$, $\mathrm{v}_{2}\{4\}$ and $\mathrm{v}_{2}\{6\}$ vs $\mathrm{N}_{\text {trk }}^{\text {offline for }}$ charged particles at $13 \mathrm{TeV}$ pp collisions [12]. Middle (right): The $\mathrm{v}_{2}\{2\}, \mathrm{v}_{2}\{4\}, \mathrm{v}_{2}\{6\}$, $\mathrm{v}_{2}\{8\}$, and $\mathrm{v}_{2}\{\mathrm{LYZ}\}$ at $5 \mathrm{TeV}$ $\mathrm{pPb}$ (2.76 TeV PbPb) collisions [11]. The statistical (systematic) uncertainties are denoted with the error bars (shaded areas).

In order to further investigate possible collective behavior in pp collisions, the $\mathrm{v}_{2}$ coefficients are also extracted from the correlations between the strange, $\mathrm{K}_{\mathrm{S}}^{0}$ and $\Lambda / \bar{\Lambda}$, and charged hadrons. The upper panel of figure 4 shows the $v_{2}$ values of charged hadrons, $\mathrm{K}_{\mathrm{S}}^{0}$ mesons and $\Lambda / \bar{\Lambda}$ hyperons as a function of $\mathrm{p}_{\mathrm{T}}$. At small- $\mathrm{p}_{\mathrm{T}}$, a mass ordering in the $\mathrm{v}_{2}$ is seen in pp collisions. In the bottom panel of figure 4 are presented $\mathrm{v}_{2}$ values scaled to the number of constituent quarks, $\mathrm{n}_{\mathrm{q}}$, plotted $v s$ scaled kinetic transverse energy, $\mathrm{KE}_{\mathrm{T}} / \mathrm{n}_{\mathrm{q}}$, which shows that similarly as in the case of $\mathrm{PbPb}$ and $\mathrm{pPb}$ [13] collisions, a collective behavior on partonic level is present also in pp collisions at $13 \mathrm{TeV}$ [12].

Due to the initial-state fluctuations, the factorization of the $\mathrm{V}_{\mathrm{n} \Delta}$ into a product of single-particle anisotropies does not hold precisely. A new observable, $r_{n}$ (more details in [14-16]) is introduced. It is defined as $\mathrm{r}_{\mathrm{n}}=\frac{\mathrm{V}_{\mathrm{n} \Delta}\left(\mathrm{p}_{\mathrm{T}}^{\mathrm{a}}, \mathrm{p}_{\mathrm{T}}^{\mathrm{b}}\right)}{\sqrt{V_{n \Delta}\left(p_{T}^{a}, p_{T}^{a}\right)} \sqrt{V_{n \Delta}\left(p_{T}^{b}, p_{T}^{b}\right)}}$ which is approximately equal to the $\cos \left[\mathrm{n}\left(\Psi_{\mathrm{n}}\left(\mathrm{p}_{\mathrm{T}}^{\mathrm{a}}\right)-\Psi_{\mathrm{n}}\left(\mathrm{p}_{\mathrm{T}}^{\mathrm{b}}\right)\right)\right]$. If the $r_{n}$ is smaller (equal) than 1 then factorization breaks (holds). The value greater than 1 means that there are unremoved non-flow effects. In figure 5 are shown magnitudes of the factorization breaking effect over a wide centrality/multiplicity range. In the case of the 2 nd harmonic, the smallest effect is observed for semi-central collisions. Going to more central or more peripheral collisions the size of 

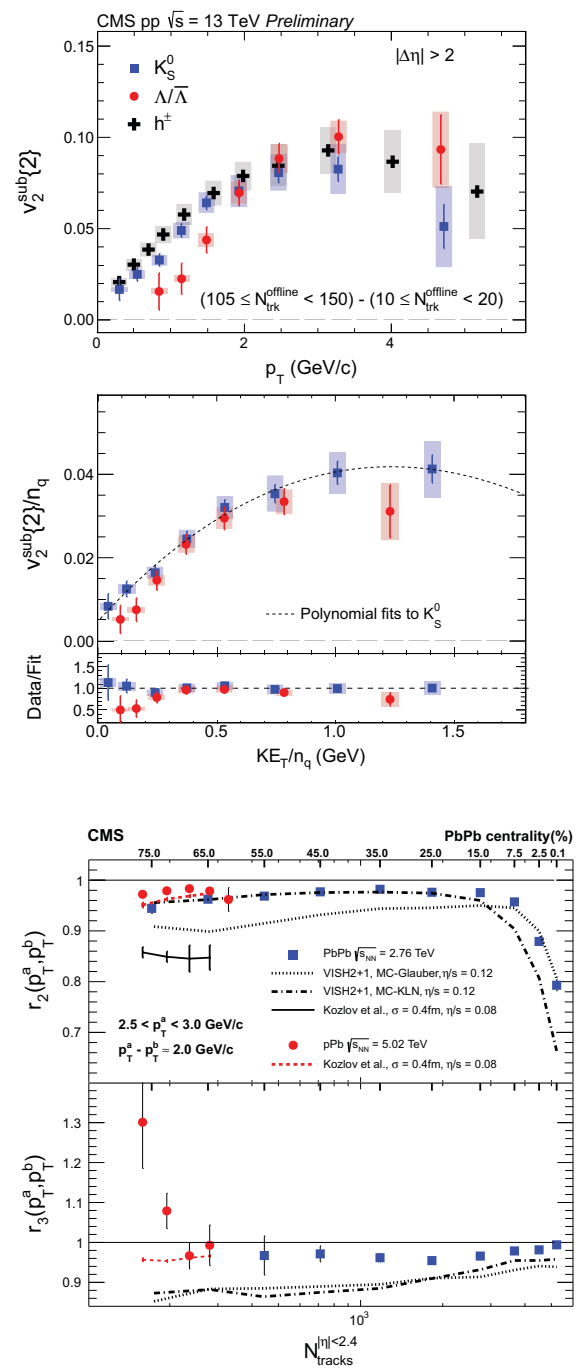

Figure 4. Top: the $\mathrm{v}_{2}\left(\mathrm{p}_{\mathrm{T}}\right)$ for $\mathrm{K}_{\mathrm{S}}^{0}$ (blue squares), $\Lambda / \bar{\Lambda}$ (red circles), and charged particles (black crosses) for the multiplicity range $105 \leq \mathrm{N}_{\mathrm{trk}}^{\text {offline }}<150$ in $13 \mathrm{TeV}$ pp collisions. Middle: the $\mathrm{n}_{\mathrm{q}}$-scaled $\mathrm{v}_{2}$ values of $\mathrm{K}_{\mathrm{S}}^{0}$ and $\Lambda / \bar{\Lambda}$ particles as a function of $\mathrm{KE}_{\mathrm{T}} / \mathrm{n}_{\mathrm{q}}$. Bottom: Ratios of $\mathrm{v}_{2} / \mathrm{n}_{\mathrm{q}}$ to a smooth fit function of $\mathrm{v}_{2} / \mathrm{n}_{\mathrm{q}}$ for $\mathrm{K}_{\mathrm{S}}^{0} v s \mathrm{KE}_{\mathrm{T}} / \mathrm{n}_{\mathrm{q}}$ [12]. The error bars correspond to statistical, while the shaded areas to systematic uncertainties.
Figure 5. The factorization ratios, $\mathrm{r}_{2}$ and $\mathrm{r}_{3}$, as a function of event multiplicity in $\mathrm{pPb}$ and $\mathrm{PbPb}$ collisions [16]. The curves show few theoretical predictions for $\mathrm{PbPb}$ and $\mathrm{pPb}$ collisions. The horizontal solid lines denote the $r_{2}$ (top) and $r_{3}$ (bottom) value of unity. The error bars correspond to statistical uncertainties, while systematic uncertainties are negligible, and thus are not shown.

the effect increases. At the same multiplicity, the size of the effect is rather similar in the two colliding systems. In $\mathrm{PbPb}$ collisions, the size of the $\mathrm{r}_{3}$ is small and nearly independent of centrality, while in $\mathrm{pPb}$ collisions the $\mathrm{r}_{3}$ approaches to 1 and then goes significantly above 1 at lower multiplicities. The $\mathrm{p}_{\mathrm{T}}$-dependent data are qualitatively described by viscous hydrodynamic models with fluctuating initial-state conditions, while they are insensitive to the shear viscosity to entropy density ratio [16]. This promises of using the factorization data to disentangle contributions of the initial-state conditions and the medium's transport properties to the collective flow, and improving modeling of the evolution of the strongly-coupled quark gluon medium.

The PCA results from 5.02 TeV high-multiplicity $\mathrm{pPb}$ and $2.76 \mathrm{TeV} \mathrm{PbPb}$ collisions [19] are presented in figure 6 and 7 respectively. The extracted leading flow modes are essentially equal to the results [7, 24] obtained from two-particle correlations. A qualitatively new result obtained using the PCA method is the existence of the sub-leading mode, $\mathrm{v}_{\mathrm{n}}^{(2)}$, which is a consequence of the initialstate fluctuations. A non-zero $\mathrm{v}_{2}^{(2)}$ is found both in $\mathrm{pPb}$ (left panel in figure 6) and in $\mathrm{PbPb}$ (upper 

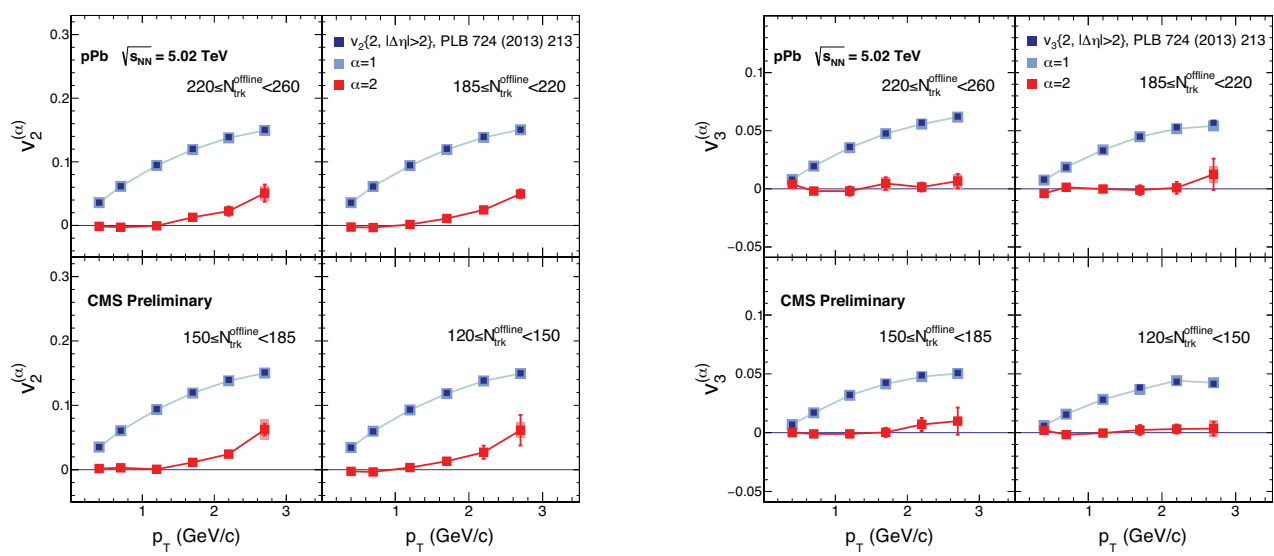

Figure 6. The first and the second mode of $\mathrm{v}_{2}\left(\mathrm{p}_{\mathrm{T}}\right)$ (left) and $\mathrm{v}_{3}\left(\mathrm{p}_{\mathrm{T}}\right)$ (right) from $5.02 \mathrm{TeV}$ high-multiplicity $\mathrm{pPb}$ collisions [19]. The $\mathrm{v}_{2}^{(1)}$ are compared to the $\mathrm{v}_{\mathrm{n}}$ harmonics measured by the CMS collaboration using the two-particle correlation method [7]. The error bars correspond to statistical while shaded areas to systematic uncertainties.

row in figure 7) collisions. It is close to zero at small- $\mathrm{p}_{\mathrm{T}}$ and achieves few percent at $\mathrm{p}_{\mathrm{T}} \approx 3 \mathrm{GeV} / \mathrm{c}$. While the strength of the effect does not depend on multiplicity in $\mathrm{pPb}$ collisions, there is a centrality dependence of the $\mathrm{v}_{2}^{(2)}$ in $\mathrm{PbPb}$ collisions. The similar behavior is established in [16]. The $\mathrm{v}_{3}^{(2)}$ is close to zero in both collision cases (right and bottom panel in figure 6 and 7) which is in an agreement with the results from [16].

\section{Summary}

In this contribution are presented results on the $v_{2}$ and $v_{3}$ measurements from the CERES at the top SPS energy, and from the CMS collaboration at the LHC energy. The CMS measured the $\mathrm{v}_{2}$ up to $100 \mathrm{GeV} / \mathrm{c}$. This result is complementary to the $\mathrm{R}_{\mathrm{AA}}$ measurements. For the first time, the $\mathrm{v}_{2}$ has been measured in pp collision at $13 \mathrm{TeV}$. This, together with the established $\mathrm{n}_{\mathrm{q}}$-scaling shows that collective behavior appears even in such small system as created in pp collision. Also, for the first time sub-leading flow modes, $\mathrm{v}_{\mathrm{n}}^{(2)}$, are measured in $\mathrm{PbPb}$ and high-multiplicity $\mathrm{pPb}$ collisions. The obtained results are in a qualitative agreement with factorization-breaking results.

\section{References}

[1] N. Borghini, P. M. Dinh, and J.-Y. Ollitrault, Phys. Rev C 64, 054901 (2001)

[2] C. Adler et al., STAR Collaboration, Phys. Rev. C 66, 034904 (2002)

[3] M. Luzum and J.-Y. Ollitrault, Phys. Rev. C 87, 044907 (2013)

[4] R. S. Bhalerao, N. Borghini, and J.-Y. Ollitrault, Nucl. Phys. A 727, 373 (2003)

[5] N. Borghini, R. S. Bhalerao, and J.-Y. Ollitrault, J. Phys. G 30, S1213 (2004)

[6] CMS Collaboration, CMS-HIN-15-014 (2015)

[7] CMS Collaboration, Phys. Lett. B 724, 213 (2013) 

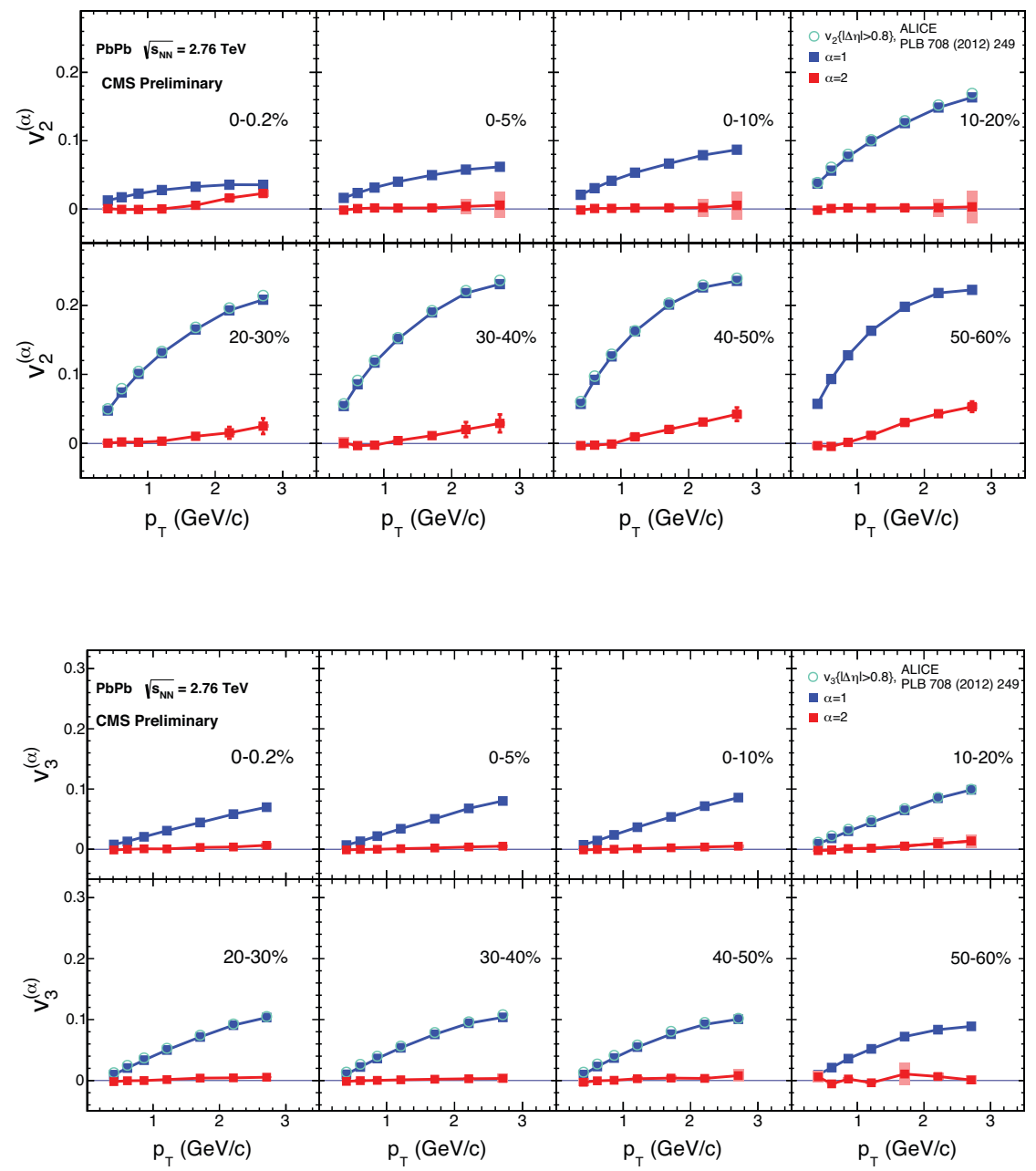

Figure 7. The first and the second mode of $\mathrm{v}_{2}\left(\mathrm{p}_{\mathrm{T}}\right)$ (upper row) and $\mathrm{v}_{3}\left(\mathrm{p}_{\mathrm{T}}\right)$ (bottom raw) from $2.76 \mathrm{TeV} \mathrm{PbPb}$ collisions [19]. The $\mathrm{v}_{2}^{(1)}$ are compared to the $\mathrm{v}_{\mathrm{n}}$ harmonics measured by the ALICE collaboration using the two-particle correlation method [24]. The error bars correspond to statistical while shaded areas to systematic uncertainties.

[8] CMS Collaboration, Phys. Lett. B 718, 795 (2013)

[9] CMS Collaboration, JHEP 09, 091 (2010)

[10] CMS Collaboration, Phys. Rev. Lett. 116, 172301 (2016)

[11] CMS Collaboration, Phys. Rev. Lett. 115, 012301 (2015)

[12] CMS Collaboration, arXiv:1606.06198, CMS-HIN-16-010 (2016)

[13] CMS Collaboration, Phys. Lett. B 742, 200 (2015)

[14] F. G. Gardim et al., Phys. Rev. C 87, 031901 (2013)

[15] U. Heinz et al., Phys. Rev. C 87, 034913 (2013)

[16] CMS Collaboration, Phys. Rev. C 92, 034911 (2015) 
[17] R. S. Bhalerao, J.-Y. Ollitrault, S. Pal, and D. Teaney, Phys. Rev. Lett. 114, 152301 (2015)

[18] A. Mazeliauskas, and D. Teaney, Phys. Rev. C 91, 044902 (2015)

[19] CMS Collaboration, arXiv:1510.03373, CMS-HIN-15-010 (2015)

[20] CMS Collaboration, JINST 3, S08004 (2008)

[21] I.A. Karpenko, P. Huovinen, H. Petersen, and M. Bleicher, Phys. Rev. C 91, 064901 (2015)

[22] D. Adamova et al., CERES NA45 Collaboration, Nucl. Phys. A 957, 99 (2017)

[23] L. Adamczyk et al., STAR Collaboration, Phys. Rev. Lett. 116, 112302 (2016)

[24] ALICE Collaboration, Phys. Lett. B 708, 249 (2012) 